This item was submitted to Loughborough's Research Repository by the author.

Items in Figshare are protected by copyright, with all rights reserved, unless otherwise indicated.

\title{
Matryoshka locally resonant sonic crystal
}

PLEASE CITE THE PUBLISHED VERSION

http://dx.doi.org/10.1121/1.3643818

PUBLISHER

(c) Acoustical Society of America

VERSION

VoR (Version of Record)

LICENCE

CC BY-NC-ND 4.0

REPOSITORY RECORD

Elford, Daniel P., Luke Chalmers, F.V. Kusmartsev, and G.M. Swallowe. 2019. "Matryoshka Locally Resonant Sonic Crystal". figshare. https://hdl.handle.net/2134/14890. 
This item was submitted to Loughborough's Institutional Repository (https://dspace.lboro.ac.uk/) by the author and is made available under the following Creative Commons Licence conditions.

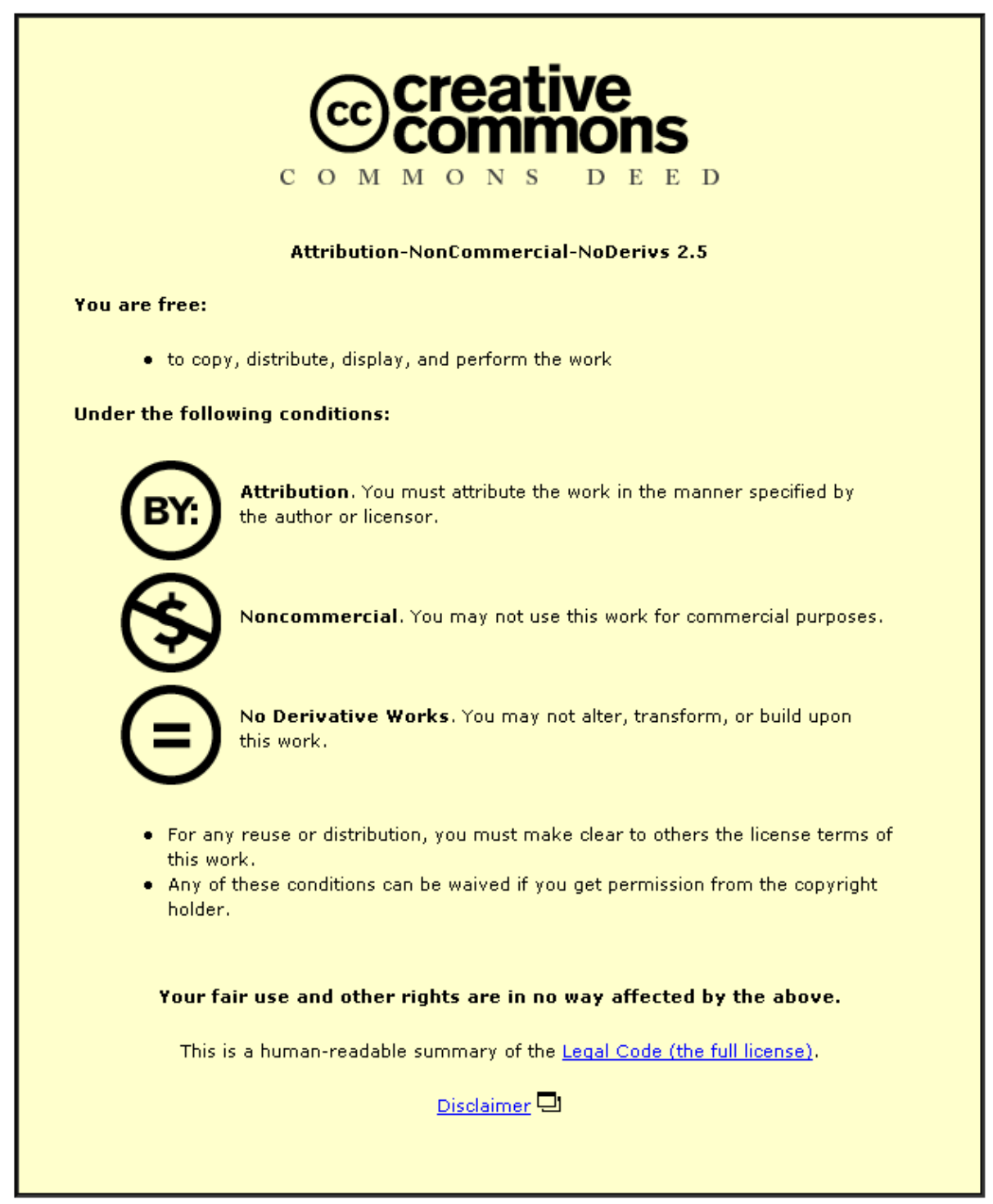

For the full text of this licence, please go to: http://creativecommons.org/licenses/by-nc-nd/2.5/ 


\title{
Matryoshka locally resonant sonic crystal
}

\author{
Daniel P. Elford, ${ }^{\text {a) }}$ Luke Chalmers, Feodor V. Kusmartsev, and Gerry M. Swallowe \\ Department of Physics, Loughborough University, Loughborough, LE11 3TU, United Kingdom
}

(Received 5 February 2011; revised 30 August 2011; accepted 3 September 2011)

\begin{abstract}
The results of numerical modeling of sonic crystals with resonant array elements are reported. The investigated resonant elements include plain slotted cylinders as well as their various combinations, in particular, Russian doll or Matryoshka configurations. The acoustic band structure and transmission characteristics of such systems have been computed with the use of finite element methods. The general concept of a locally resonant sonic crystal is proposed that utilizes acoustic resonances to form additional band gaps that are decoupled from Bragg gaps. An existence of a separate attenuation mechanism associated with the resonant elements that increases performance in the lower frequency regime has been identified. The results show a formation of broad band gaps positioned significantly below the first Bragg frequency. For low frequency broadband attenuation, a most optimal configuration is the Matryoshka sonic crystal, where each scattering unit is composed of multiple concentric slotted cylinders. This system forms numerous gaps in the lower frequency regime, below Bragg bands, while maintaining a reduced crystal size viable for noise barrier technology. The finding opens alternative perspectives for the construction of sound barriers in the low frequency range usually inaccessible by traditional means including conventional sonic crystals.
\end{abstract} (C) 2011 Acoustical Society of America. [DOI: 10.1121/1.3643818]

PACS number(s): 43.40.Fz, 43.20.Gp, 43.20.Ks, 43.25.Jh [ANN] Pages: 2746-2755

\section{INTRODUCTION}

Recent years have seen a growing interest in sonic crys$\operatorname{tals}^{1,2}$ and their potential for use as noise barriers with reported sound attenuation up to $20 \mathrm{~dB}$ (Ref. 3) and $25 \mathrm{~dB} .{ }^{4}$ Such crystals usually consist of periodic arrays of a high mechanical impedance material (often as cylindrical rods) and are known to give high attenuation at selective but often rather narrow frequency bands as a consequence of multiple scattering phenomena. An advantage of sonic crystal noise barriers is that by varying the distance between the scatterers, it is possible to attain peaks of attenuation in a selected frequency range. Further advantages of a sonic crystal barrier in comparison with more traditional solid sound barriers are its ability to allow light to pass and, uniquely, that it does not present an obstruction to the free flow of air. The relationship between the lattice parameter and operating frequency suggests extremely large barriers will be required to attenuate lower frequency noise such as traffic. Therefore locally resonant sonic materials $(\mathrm{LRSM})^{5}$ are better suited due to their ability to form band gaps decoupled from the periodicity. However, these band gaps cover a narrow attenuation range and such LRSM are unsuitable for use as a noise barrier.

We investigate the effects of elastic wave propagation through a new class of LRSM with multiple acoustic resonances, capable of broadening the range of attenuation. The proposed sonic crystal forms broad attenuation bands in the lower frequency regime and comprises concentric slotted cylinders. The preliminary results of this work are presented in Ref. 6. Previously Hu et al. ${ }^{7}$ constructed a sonic crystal

\footnotetext{
a) Author to whom correspondence should be addressed. Electronic mail: D.Elford@lboro.ac.uk
}

lens composed of an array of two-dimensional Helmholtz resonators, which in the long-wave regime was found to have a high relative acoustic refractive index $n$ and at the same time, a small acoustic impedance $Z$ mismatch with air for airborne sound. Furthermore, the wave propagation in a sonic crystal with Helmholtz resonator defect was studied by Wu et al. ${ }^{8}$ where a Helmholtz resonator is placed as a point defect of the sonic crystal and exhibits local resonance phenomena. Similar acoustic metamaterials have shown sound confinement and focusing. ${ }^{9}$ Movchan et al. investigated the asymptotic analysis of an eigenvalue problem for the Helmholtz operator in a periodic structure involving split-ring resonators and associated multistructures where the position of stop bands was deduced from an asymptotic model. ${ }^{10}$

In the present paper, an array of the resonant elements that have broad resonances below the Bragg band gaps have been studied. In particular, the elements having a shape of slotted cylinders and their various configurations have been considered. The interaction between their resonances produces band gaps and gives rise to phenomena that can lead to acoustic attenuation. The continuum band of the surrounding effective medium interacts with resonance states by hybridization (mixtures of different waves states) giving rise to hybridization gaps such as those found in three dimensional solid phononic crystals ${ }^{11}$ and experimentally in colloidal films. ${ }^{12}$ The proposed systems have been studied numerically with the use of finite elements methods (FEM) as the analytical techniques for calculating the band structure and transmission become impractical with phononic crystals of complex geometries. The complicated geometries are difficult to express by means of elementary functions. Using FEM, unusual geometries can easily be described, and the continuous problem can be discretized. ${ }^{13,14}$ To validate the numerical analysis, we have replicated the results presented 
by Movchan et al. ${ }^{10}$ Here calculations of the band structure are performed using an asymptotic approximation. The multistructure reported is comprised from two " $\mathrm{C}$ "-shaped cavities concentrically arranged. For the dimensions detailed in Ref. 10, the first two eigenvalues calculated by our FEM model are found to be $\omega_{1}=0.777$ and $\omega_{2}=1.766$. These are in good agreement with those predicted by the asymptotic approximation model thus, confirming the reliability of the FEM calculations. It should be noted that the asymptotic analysis does not hold for cavities with large slot widths, as the approximation of a thin ligament becomes invalid. It is found that the behavior of resonators with a large slot is best described by the standard formula for Helmholtz resonators ${ }^{15}$ even when arranged concentrically.

\section{NUMERICAL MODELLING OF RESONANT ARRAYS WITH THE USE OF FINITE ELEMENT METHOD}

\section{A. Eigenvalue analysis}

First we consider infinite arrays of solid cylinders. Their band structures are obtained using the FEM that was developed in the framework of COMSOL MULTIPHYSICS. ${ }^{16}$ For a sonic crystal in a two-dimensional square array, the unit cell (seen in Fig. 1) is used as a basis for the calculations. The structure is assumed to be infinite and periodic in the direction $x$ with the period $a_{1}$ and in the direction $y$ with the period $a_{2}$ and described by two basis vectors: $\left(a_{1}, 0\right)$ and $\left(0, a_{2}\right)$. According to the Floquet-Bloch theorem, the relation for the pressure distribution $p$ for nodes lying on the boundary of the unit cell can be expressed as

$$
p\left(\vec{x}+\vec{a}_{1}+\vec{a}_{2}\right)=p(x) \exp \left[i\left(k_{x} a_{1}+k_{y} a_{2}\right)\right],
$$

where $x$ is the position vector in the unit cell and $\vec{k}=\left(k_{x}, k_{y}\right)$ is the Bloch wavevector. Considering the periodic boundary conditions in the preceding text allows the reduction of the model to a single unit cell. First we apply boundary conditions of the Neumann type; this is required on boundaries where pressure $p$ is controlled by a periodic boundary condition. Next a phase relation is applied in the boundary of the unit to define boundary conditions between adjacent units. This phase relation is related to the wavenumber of the incident wave in the periodic structure. The periodic boundary conditions are applied to truncate the twodimensional simulation plane in the $x$ and $y$ directions by reducing the system to one unit cell. An ideal crystal is infinitely periodic, hence the periodic boundary condition ensures that the finite simulation space mimics an infinitely periodic crystal in the $x$ and $y$ directions. The pressure components at all edges of the computational domain are relocated by the periodic boundary conditions to the opposite edges of the domain. This enforces the condition that a wave travelling into the top edge of the computational domain is relocated and appears outside the computational domain in the bottom periodic boundary condition. Similarly a wave travelling into the bottom edge of the domain is relocated and appears outside the computational domain in the top periodic boundary condition domain. Similarly this occurs for the left and right edges of the domain. The cylinder in the unit cell is consid-

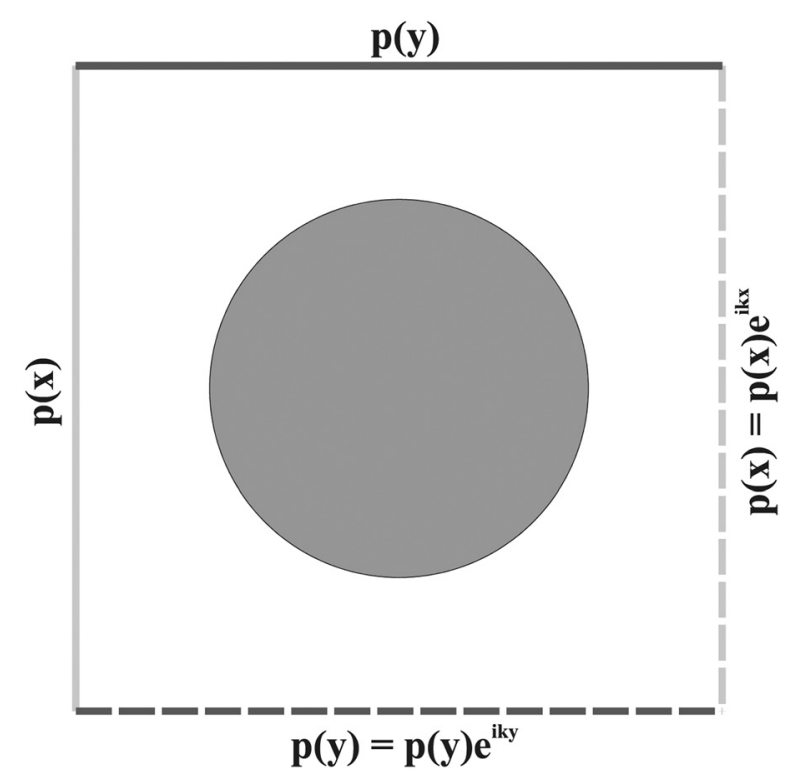

FIG. 1. Single unit cell of an infinite sonic crystal system with FloquetBloch boundary conditions applied to the borders of the unit cell.

ered to be rigid, and therefore the Neumann boundary condition is applied to its surface. By defining the Bloch wavevector in the first Brillouin zone, for the $\Gamma X$ direction, $k_{x}$ varied from 0 to $\pi$, whilst $k_{y}=0 ; \Gamma M$ direction $k_{y}$ varied from 0 to $\pi$, while $k_{x}=\pi$; and in the $X M$ direction $k_{x}$ and $k_{y}$ varied from 0 to $\pi$. The analysis of the first 10 eigenfrequencies and the corresponding eigenvectors is computed. The eigenvectors are related to the pressure distribution of the mode. In this investigation, the infinite sonic crystal is composed of steel cylinders in air, with lattice parameter $a=22$ $\mathrm{mm}$, radius of steel scatterer $r=6.5 \mathrm{~mm}$, and packing fraction $f=0.27$. Figure 2 displays the characteristic band structure for this system and is plotted in the three principal symmetry directions.

The dispersion remains isotropic in the low-frequency range, following a linear trend $c=\omega / k$, where the propagating wave cannot resolve the fine structure of the cylinders in the long-wavelength limit. A sonic band gap opens between the first two bands in the $\Gamma X$ direction. It can be seen that toward the edges of the Brillouin zone, the dispersion is no longer linear with a curving of the bands where, at the edge, the bands exhibit zero group velocity.

\section{B. Transmission analysis}

The finite element method has been utilized to calculate the pressure field behind a sonic crystal and to generate a pressure map of the system at fixed frequencies. The COMSOL MULTIPHYSICS software is adopted to solve the acoustic wave propagation in the sonic crystals. The equation used to analyze the acoustic wave problems is expressed as

$$
\frac{1}{\rho_{0} c^{2}} \frac{\partial^{2} p}{\partial t^{2}}+\nabla \cdot\left(-\frac{1}{\rho_{0}} \nabla p\right)=0
$$

This reduces to a Helmholtz equation for a time harmonic pressure wave excitation, $p=p_{0} e^{i \omega t}$, 


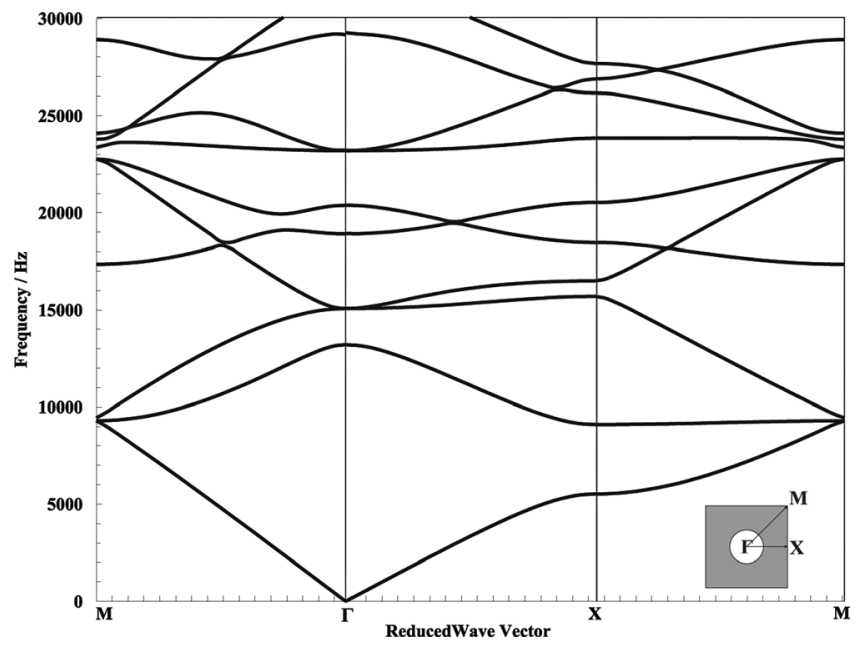

FIG. 2. Finite element computed band structure for a sonic crystal consisting of steel scatterers embedded in air $(r=6.5 \mathrm{~mm}, a=22 \mathrm{~mm})$. Inset: Brillouin zone. $\Gamma X$ refers to the $\left[\begin{array}{ll}1 & 0\end{array}\right]$ direction, and $\Gamma M$ the $\left[\begin{array}{ll}1 & 1\end{array}\right]$ direction, while $X M$ refers to the wavevector varying from [1 0$]$ to [1 1 1] on the side of the Brillouin zone.

$$
\nabla \cdot\left(-\frac{1}{\rho_{0}} \nabla p_{0}\right)-\frac{\omega^{2} p_{0}}{\rho_{0} c^{2}}=0
$$

where $\omega=2 \pi f$ is the angular frequency. By solving Eq. (3), the pressure field can be obtained.

A two-dimensional sonic crystal system in a $10 \times 10$ square lattice is described in COMSOL MULTIPHYSICS with lattice parameter $a=22 \mathrm{~mm}$ and cylinder radius $r=6.5 \mathrm{~mm}$. Material parameters for this system are $\rho_{s}=7800 \mathrm{~kg} \mathrm{~m}^{-3}$, $c_{s}=6100 \mathrm{~m} \mathrm{~s}^{-1}, \rho_{a}=1.25 \mathrm{~kg} \mathrm{~m}^{-3}, c_{a}=343 \mathrm{~m} \mathrm{~s}^{-1}$ where subscripts denote the air domain and steel scatterers. In the case of the rigid cylinders in the sonic crystal system, soundhard boundary conditions have been applied; i.e., the normal component of the velocity of the air particles is zero in the walls of the cylinders. The radiation boundary conditions at the exterior edges of the rectangular domain are considered to be perfectly absorbing. In the simulations, a rising tone noise source at the left edge of the domain, from 0 to 30000 $\mathrm{Hz}$, is modeled as a radiation condition with pressure source set to $1 \mathrm{~Pa}$, which is equivalent to a $90 \mathrm{~dB}$ source. For the numerical simulation, we use a triangular mesh of approximately $10^{6}$ elements, with at least 10 elements per wavelength to solve the wave equation across the domain.

The illustrated pressure maps are taken at $4000 \mathrm{~Hz}$ (pre Bragg band gap formation), $8000 \mathrm{~Hz}$ (in the center of the band gap), and $12000 \mathrm{~Hz}$ (after Bragg band gap formation) as shown in Fig. 3. The pressure map taken pre band gap formation demonstrates that at low frequencies the sonic crystal system behaves as a homogeneous material and acoustic wave propagation is unaffected by the periodic structure. This is due to the lattice parameter being much smaller than the relevant wavelength. The pressure map at $8000 \mathrm{~Hz}$, in the center of the band gap shows band gap formation with the wavelength of the incoming acoustic wave comparable to the lattice parameter. The acoustic wave is severely attenuated due to multiple scattering effects and a shadow zone is formed behind the sonic crystal. At $12000 \mathrm{~Hz}$, post band gap

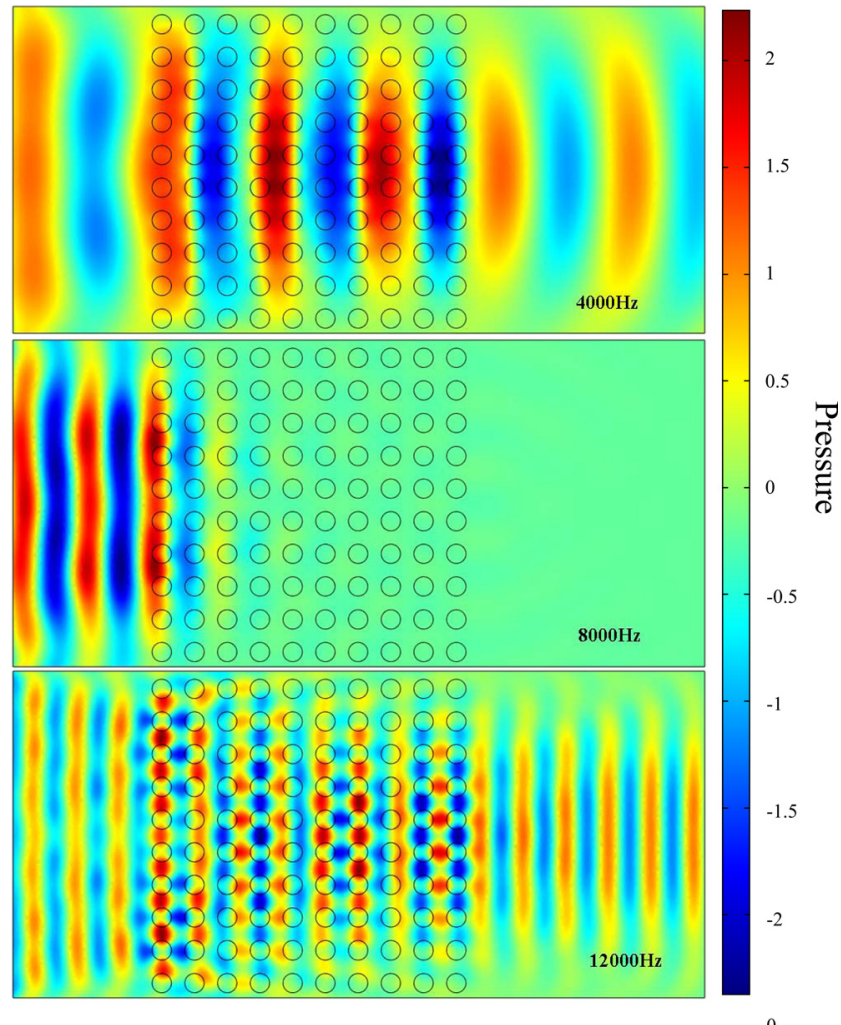

FIG. 3. (Color online) Finite element computed pressure maps for solid steel cylinders in air taken at three frequencies: 4000, 8000, and $12000 \mathrm{~Hz}$.

formation, the wavelength of the acoustic wave is smaller than that of the lattice parameter of the sonic crystal system. The wave is free to propagate through the sonic crystal system as the plane wave cannot resolve the individual scatterers.

By solving for a parametric sweep of frequency, a frequency spectrum displaying the attenuation properties of the sonic crystal can be constructed. A comparison of the
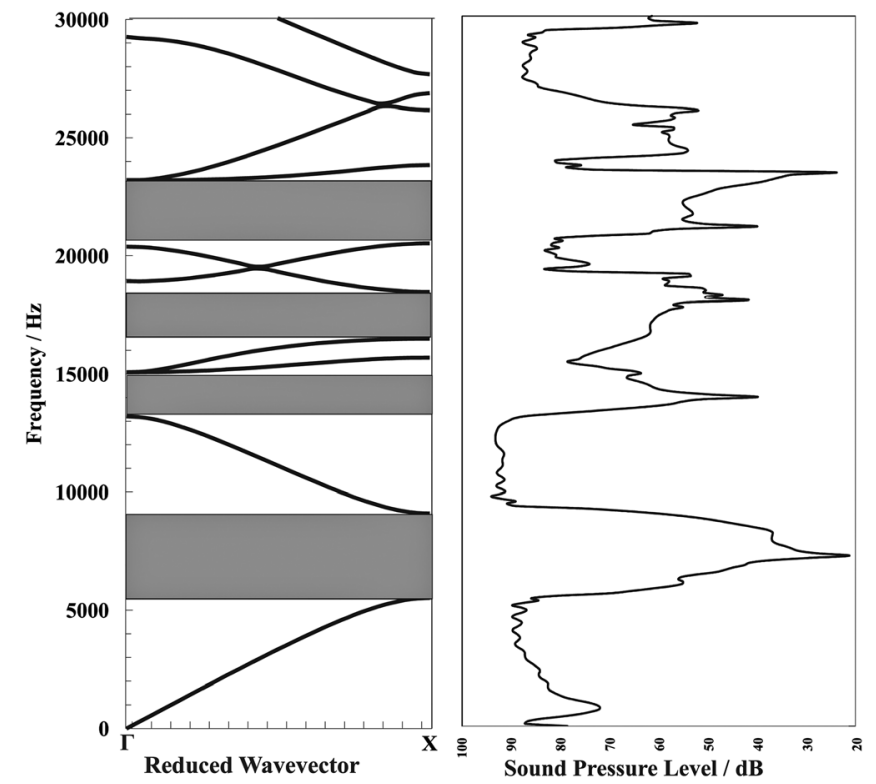

FIG. 4. A comparison of finite element computed band structure in the $\Gamma X$ direction against the finite element computed frequency spectra for sound pressure level for conventional sonic crystal. 
transmission spectrum from 0 to $30000 \mathrm{~Hz}$ against the computed band structure, limiting the study to the $\Gamma X$ direction can be seen in Fig. 4. The finite element transmission calculations give band gaps of larger width than those predicted by the band structure. This difference can be attributed to the finite number of scatterers used in the transmission simulations and the subsequent diffraction effects around the edges of the sonic crystal structure. The overall position of the band gaps are in good agreement with those of the band structure calculations.

\section{C-SHAPED LOCALLY RESONANT SONIC CRYSTAL}

The conventional sonic crystal modeled with solid scattering inclusions forms band gaps solely due to the periodicity in agreement with theory and our experiments. ${ }^{6}$ To operate below this Bragg gap, we now investigate a design of a locally resonant sonic crystal (LRSC), which is an array of slotted cylinders. An advantage of using COMSOL MULTIPHYSICS to compute the acoustic band structure is the capability of modeling more complex scatterer geometries. Similar to the conventional sonic crystal system modeled previously, periodic boundary conditions have been employed, see Fig. 5 .

Again, by varying the wavevector in the first Brillouin zone for the first 10 eigenvalues, the band structure can be constructed, see Fig. 6. The figure gives the computed band structure of a two-dimensional sonic crystal, comprising slotted tubes with inner radius $5 \mathrm{~mm}$, external radius 6.5 $\mathrm{mm}$, and slot width $4 \mathrm{~mm}$ arranged in a square lattice in air. The period is $22 \mathrm{~mm}$. We call each resonating inclusion a C-shaped resonator.

We note the appearance of a flat band in the band structure (Fig. 6). Modes associated with a flat band should have a group velocity equal to zero and exhibit strong spatial localization. In practice, such localized modes are often created by inserting a defect in a periodic structure, i.e., creating

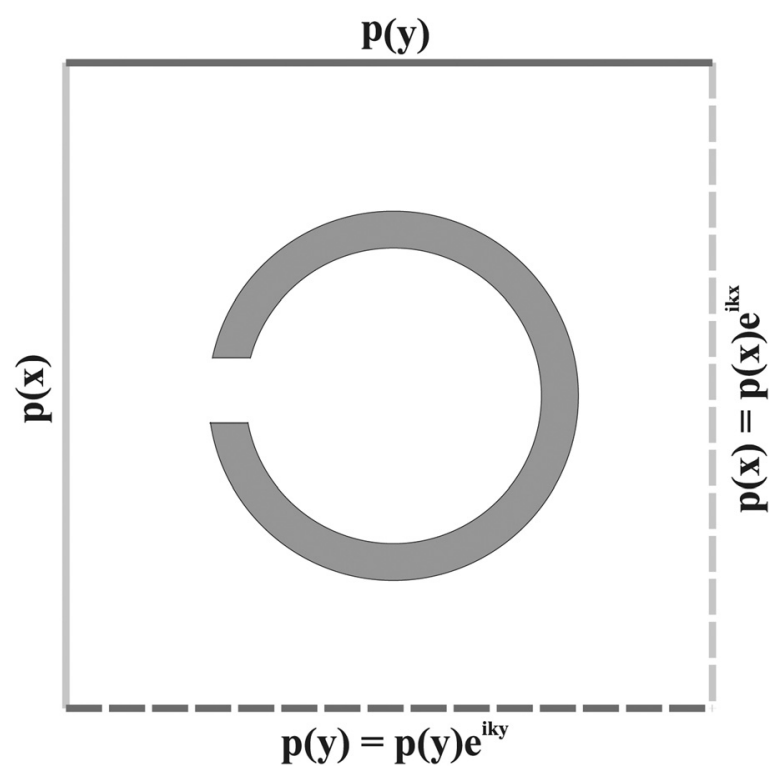

FIG. 5. Unit cell for a C-shaped locally resonant sonic crystal with FloquetBloch boundary conditions described.

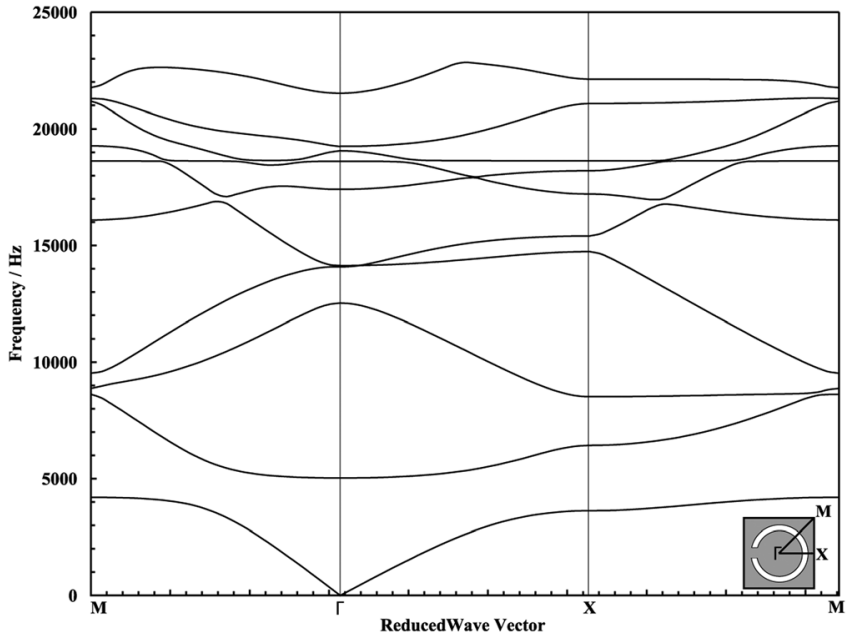

FIG. 6. Finite element computed band structure for a C-shaped locally resonant sonic crystal.

a cavity. ${ }^{8}$ It is clear that the acoustic resonance owing to the $\mathrm{C}$-shaped inclusions leads to the appearance of this flat band, forming a complete acoustic band gap that is induced by the local acoustic resonance of each individual scatterer. The slotted tubes act analogous to Helmholtz resonators and all have the same resonance frequency, predicted by a modified resonator equation (Ref. 15) $f_{\text {res }}=4840 \mathrm{~Hz}$. The combined action of the resonators induces the degenerate state to form a band gap symmetrically around $f_{\text {res }}$ spanning 4190-5190 $\mathrm{Hz}$, centered at $4690 \mathrm{~Hz}$.

Due to the periodicity of the C-shaped LRSC, the structure still exhibits Bragg band gaps in the $\Gamma X$, the first of which spans $6410-8550 \mathrm{~Hz}$. A further three Bragg bands are present due to the fulfillment of the Bragg condition located at $12525-14135 \mathrm{~Hz}, 15400-17210 \mathrm{~Hz}$, and $21050-22140 \mathrm{~Hz}$. The introduction of the extra, flat resonance band could lead to the construction of viable acoustic barriers in the low frequency regime, which offer sound attenuation in all crystal lattice planes. The flat band (originating from the localized acoustic resonance seen in the band structure) is a large anticrossing gap; this is generally referred to as a hybridization gap in the context of sonic crystals. ${ }^{11}$

FE transmission simulations are implemented for the Cshaped LRSC. Similar boundary conditions have been applied as for the conventional sonic crystal investigation. Effectively this new system is a duplicate of the conventional sonic crystal system detailed in the preceding text but with the inclusion of a slot to create a resonant cavity.

Computed pressure maps, taken at four frequencies of interest, demonstrate the propagation of an acoustic plane wave through the C-shaped LRSC. Similar to the conventional sonic crystal, at frequencies below the active frequency ( 3000 $\mathrm{Hz}$ ), the incoming wave propagates as if the system was a homogeneous medium. At $4850 \mathrm{~Hz}$, the computed pressure map shows that the C-shaped LRSC attenuates the wave in this region. The pressure map, see Fig. 7, indicates that regions of maximum pressure are localized to the inclusions at the resonance frequency. Above the resonant frequency around 6500 $\mathrm{Hz}$, the acoustic wave is free to propagate through the system. As we approach the Bragg band gap frequency of $9000 \mathrm{~Hz}$, 


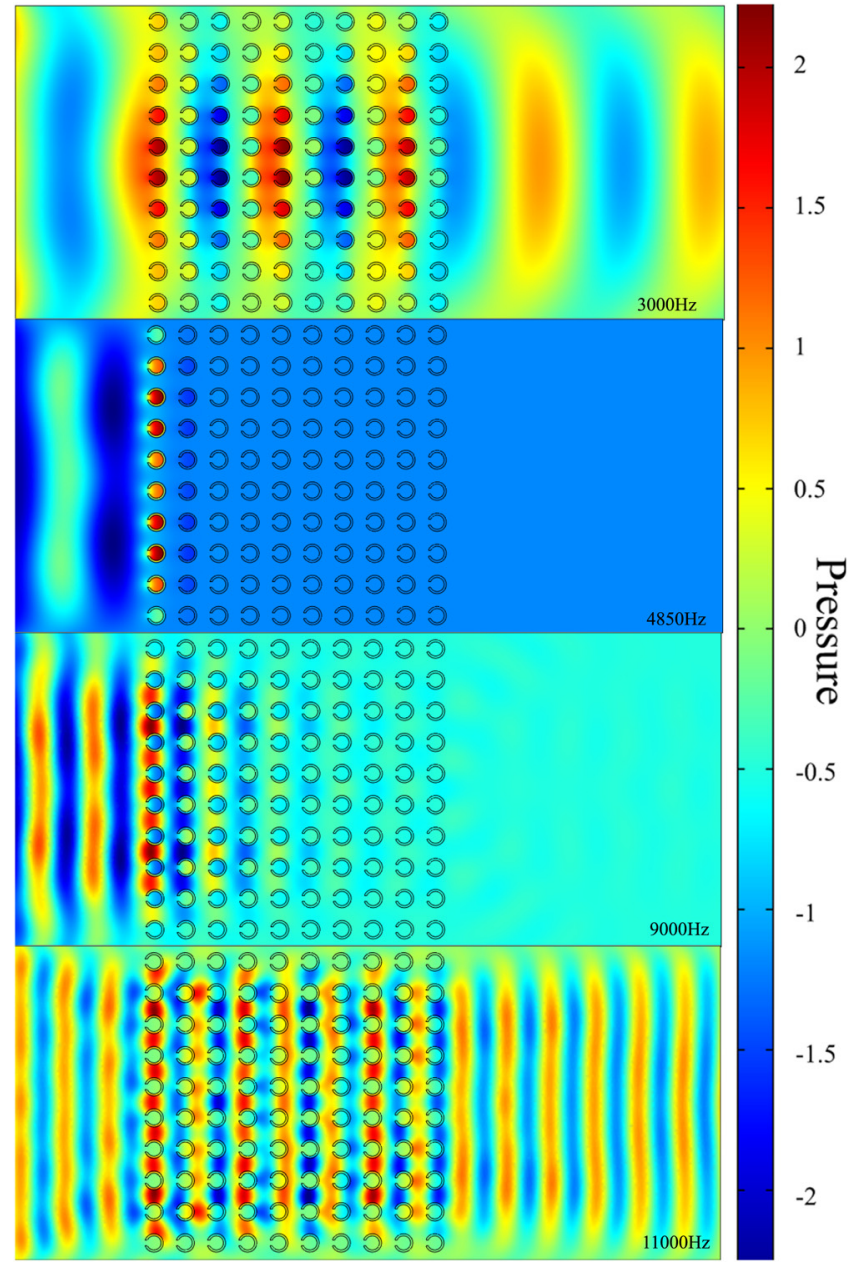

FIG. 7. (Color online) Finite element computed pressure level maps for a C-shaped locally resonant sonic crystal at 3000, 4850, 9000, and $11000 \mathrm{~Hz}$.

again we see the appearance of band gap attenuation. Looking at the pressure maps, it becomes apparent that the two regions of attenuation are controlled by two different mechanisms; resonance and Bragg scattering.

In the transmission spectrum, see Fig. 8, additional attenuation peaks can be observed $(\sim 20000 \mathrm{~Hz})$. If we compare the location of these peaks with the computed band structure, the peaks can be attributed to anticrossing regions present in the band structure. In general, such gaps originate from level repulsion when two bands of the same symmetry avoid crossing each other. The appearance of these anticrossing regions are beyond the scope of this investigation but should be investigated further to enhance the performance of the "C"-shaped LRSC. The reader is directed toward a seminal paper by $\mathrm{Wu}$ et al. ${ }^{17}$ detailing this phenomenological effect. The physical origin of these anticrossing gaps is different when compared with those induced by the acoustic resonance. The flat band (hybridization gap) originating from the acoustic resonance of the $\mathrm{C}$-shape scatterer and regions corresponding to the anticrossing gaps that are formed due to the longitudinal displacement field in the homogeneous effective medium. ${ }^{11}$ The narrowness of the anticrossing gaps indicate that they are much weaker. The hybridization discussed is analogous to s-d hybridization in the energy band structure of transition metals, see, for example, Harrison. ${ }^{18}$
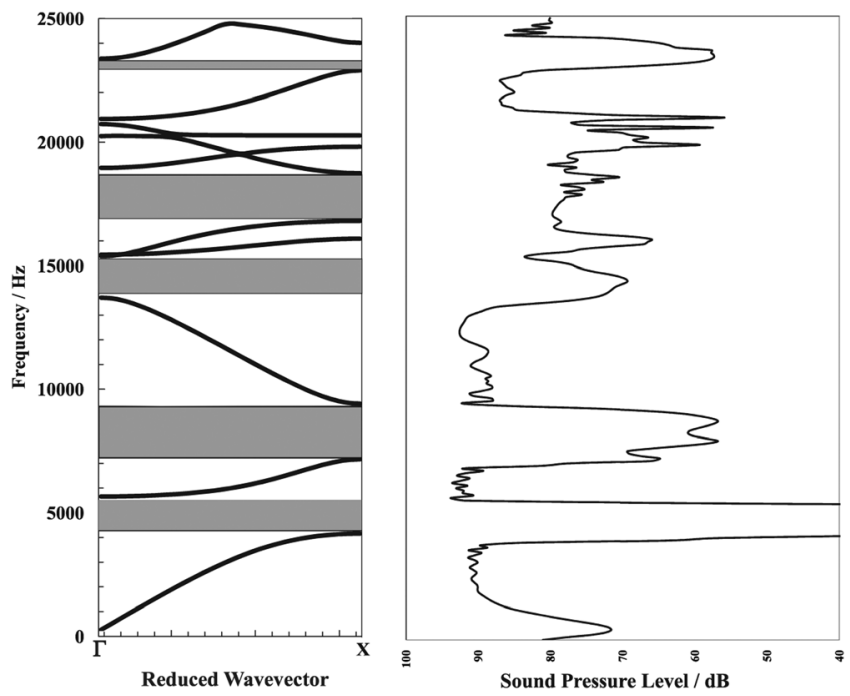

FIG. 8. A comparison between finite element computed band structure and finite element transmission simulation for a C-shaped locally resonant sonic crystal.

\section{MATRYOSHKA SONIC CRYSTAL}

The C-shaped tubes act as acoustic resonators that give rise to a single flat band that extends across all high symmetry directions and is located below the Bragg gap. Its position is dependent upon the cavity dimensions and is independent of the sonic crystal periodicity. For practical applications of sonic crystals as noise barriers, it is desirable to be able to broaden the width of this resonance gap. One method to achieve this is to include multiple resonator sizes and "overlap" the individual resonance peaks. We have investigated mixed arrays that display this ability; ${ }^{6}$ however, to save space and reduce the overall barrier thickness, we now propose a design of sonic crystal with resonators placed concentrically inside one another, extending the multistructure describe by Movchan et al. ${ }^{10}$ We coin this the Matryoshka (Russian doll) configuration. Specifically we investigate a

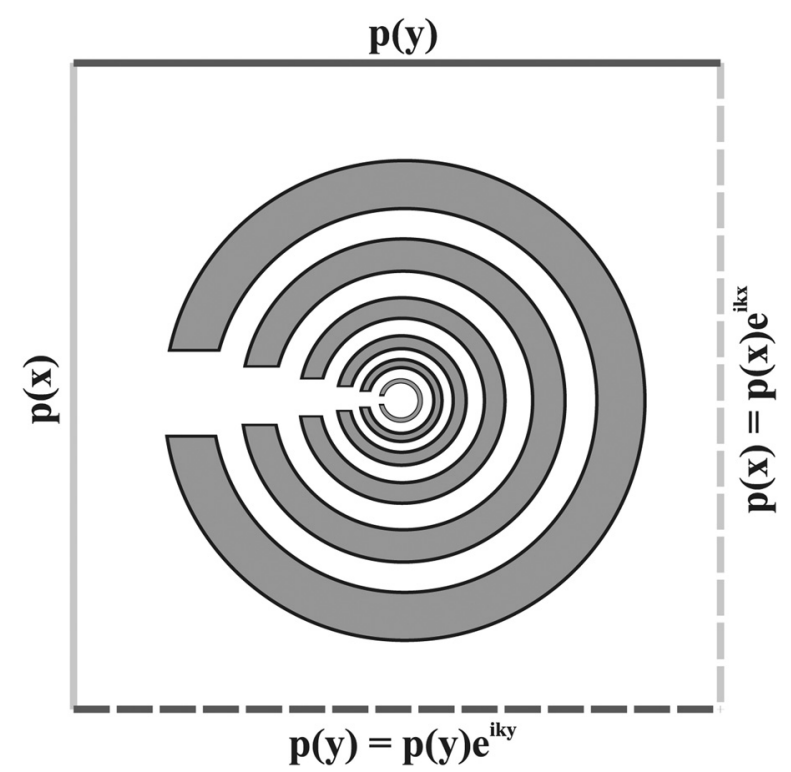

FIG. 9. Schematic of the unit cell used in band structure calculations for the six concentric Matryoshka system. 


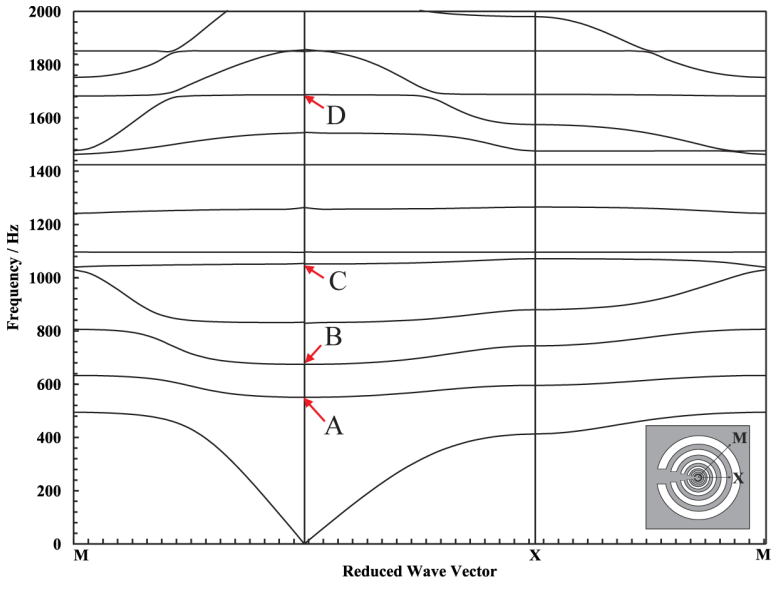

FIG. 10. (Color online) Finite element computed band structure for the six concentric Matryoshka system. (A) to (D) correspond to the eigenmode pressure distribution in Fig. 11.

Matryoshka sonic crystal the unit cell of which is defined with six concentric $\mathrm{C}$-shaped resonators, all tuned to frequencies that lie within $200 \mathrm{~Hz}$ of each other in the low frequency regime. This is achieved by increasing the dimensions of the resonating inclusions and lattice parameter (see Fig. 9).

Applying periodic boundary conditions, to replicate an infinite array of these Matryoshka inclusions in a square array with lattice parameter $a=15.5 \mathrm{~mm}$, the acoustic band structure can be computed. The dimensions of each Cshaped resonator are designed so that they can be placed concentrically inside each other. The largest C-shaped reso- nator has an external diameter $=132 \mathrm{~mm}$ and an internal diameter $=109 \mathrm{~mm}$ with a slot width $31 \mathrm{~mm}$. Subsequent concentric resonators have a scale factor of $1 / 1.3$, giving the smallest of the nested resonators an external diameter $=22.5$ $\mathrm{mm}$ and an internal diameter $=14.1 \mathrm{~mm}$ with a slot width $11.3 \mathrm{~mm}$. Figure 10 presents the finite element computed band structure in all high symmetry directions.

The band structure has been computed in the low frequency regime $<2000 \mathrm{~Hz}$, corresponding to the first 13 eigenvalues, by varying the wave vector in the first Brillouin zone. It can be seen that a Matryoshka system, with many individual resonating units, induces the formation of multiple band gaps. Due to the periodic nature of these inclusions, this sonic crystal system possesses the characteristic Bragg band gaps, although it is hard to identify which bands are attributed to the separate band gap formation mechanisms from the band structure alone. A conventional sonic crystal system with a lattice parameter $a=15.5 \mathrm{~mm}$ should possess a Bragg band gap around $1120-1360 \mathrm{~Hz}$, therefore the other band gaps present in the band structure must be caused by the acoustic resonance of each $\mathrm{C}$-shaped inclusion. It can be seen that the induced resonance band gaps are complete acoustic band gaps, inhibiting wave propagation across all lattice planes without the need for a large packing fraction as found with the characteristic Bragg band gap.

For completeness, finite element methods have been employed to obtain a transmission spectrum for this array, see Fig. 12. A $10 \times 10$ array of the Matryoshka inclusions (each containing six concentric C-shaped resonators) is described in comsol. The spectrum extends to $2000 \mathrm{~Hz}$ and
A)

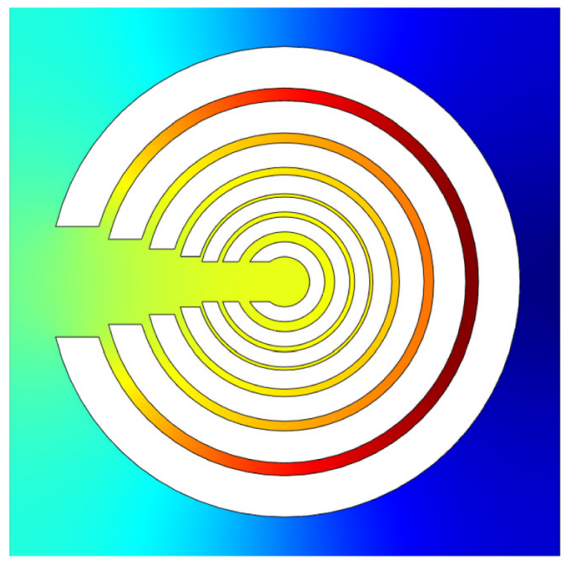

C)

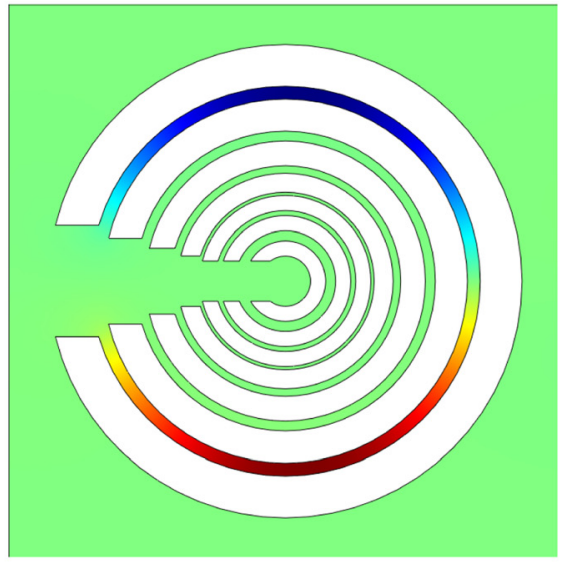

B)

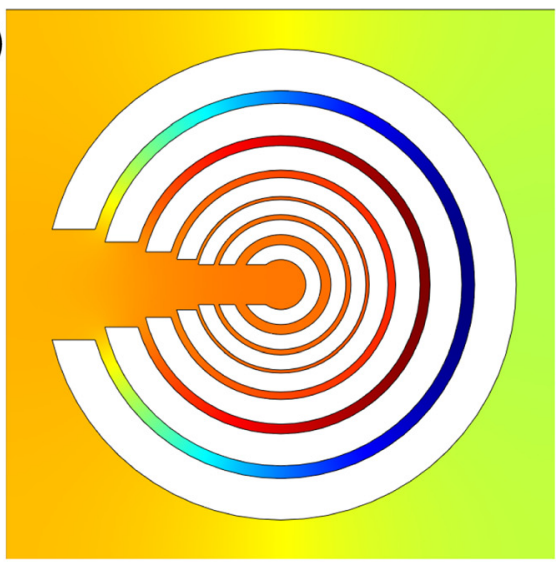

D)

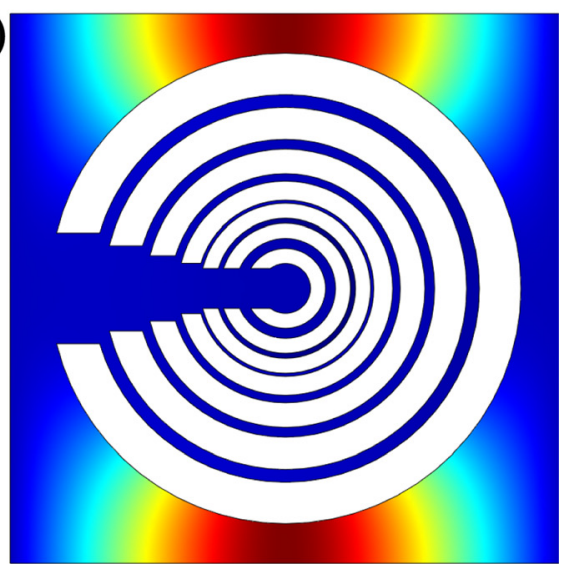

FIG. 11. (Color online) Finite element computed eigenmode pressure distribution: (A) indicating individual resonance of largest resonator, (B) second largest resonator, (C) multiple harmonic resonance of largest resonator, and (D) propagating mode, indicating a Bragg gap edge. 
TABLE I. Band gap location in comparison with Helmholtz predicted resonances.

\begin{tabular}{lcc}
\hline \hline Band Number & FEM Eigenfrequency $(\mathrm{Hz})$ & Helmholtz Eq. $(\mathrm{Hz})$ \\
\hline 1 & 550.8 & 546.1 \\
2 & 674.8 & 707.3 \\
3 & 828.9 & 925.5 \\
4 & 1051.5 & Bragg \\
5 & 1096.6 & 1092.2 \\
6 & 1263.5 & 1201.1 \\
7 & 1424.1 & 1414.5 \\
8 & 1546.0 & 1502.8 \\
9 & 1686.8 & 1638.2 \\
10 & 1850.2 & Bragg \\
11 & 1857.5 & 1851.9 \\
12 & 2106.7 & 2111.5 \\
\hline \hline
\end{tabular}

demonstrates the appearance of multiple regions of attenuation, owing to the individual resonances of the six C-shaped resonators as well as a Bragg band gap. The first attenuation band is caused by the individual resonance of the largest diameter resonating inclusion, spanning 400-600 Hz. Five more regions of attenuation can be seen spanning, 600-740 $\mathrm{Hz}, 740-880 \mathrm{~Hz}, 880-1120 \mathrm{~Hz}, 1120-1360 \mathrm{~Hz}$, and $1360-1500 \mathrm{~Hz}$.

The fact that we have large slot sizes that are aligned concentrically with the same orientation means that the asymptotic model in Ref. 10 does not hold for our alternative structure. We can use a modified Helmholtz resonator equation (Ref. 15) to predict the location of each resonance gap attributed to the different sized inclusion. A comparison of the band gap locations from finite element computation to the modified Helmholtz resonantor equation can be seen in Table I showing good agreement.

Figure 11 presents the corresponding eigenmode pressure diagrams computed for the first two bands [Figs. 11(A) and 11(B), respectively]. It can be seen that each individual resonator experiences an increase in pressure inside the cavity, caused by the acoustic resonance of each C-shaped
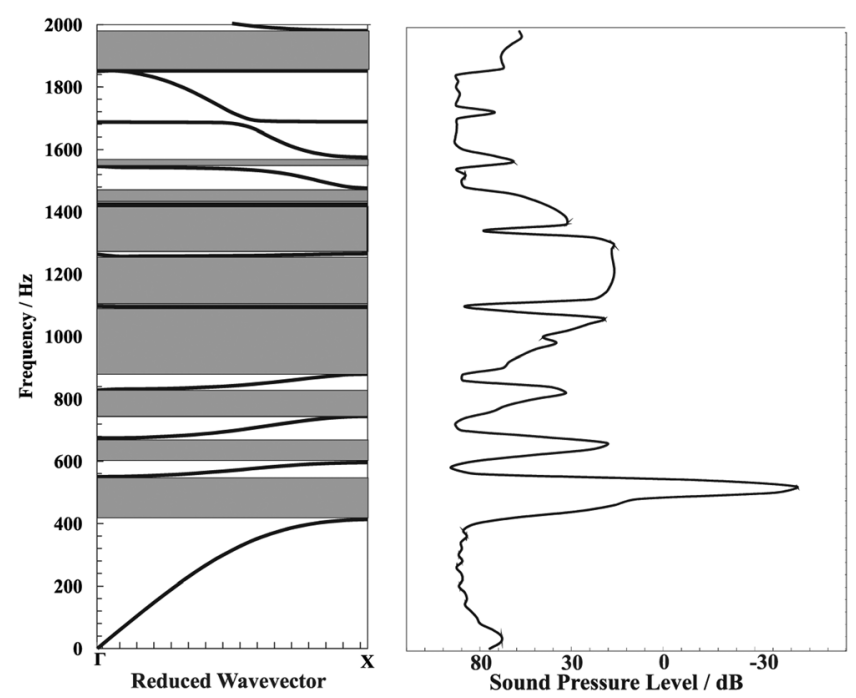

FIG. 12. A comparison of the finite element computed band structure with the finite element computed frequency spectrum for a six concentric Matryoshka system. inclusion. It should be noted that multiple harmonic resonance gaps are formed [shown in Fig. 11(C)] and induce the formation of extra gaps in the band structure. We can confirm the existence of a Bragg gap by again studying the eigenmode pressure distribution [Fig. 11(D)]. It is clear to see that this band is attributed to a propagated mode. This allows us to confirm the band gap formation mechanism, either resonance or Bragg, that is responsible for each region of attenuation present in the frequency spectrum.

For comparison, Fig. 12 shows both the finite element computed band structure, limited to the $\Gamma X$ direction, and the computed frequency spectrum. The frequencies at which the band gaps occur in the band structure are in good agreement with the regions of attenuation present in the transmission spectrum. A small attenuation band is present in the transmission spectrum at around $1700 \mathrm{~Hz}$. At the corresponding frequency in the band structure, an anticrossing region appears, induced by the level repulsion effect. Because the resonances are very close in frequency to the frequency that satisfies the Bragg condition, the two band gap regimes appear to overlap in this Matryoshka sonic crystal. Resonance scattering occurring in the same frequency range as Bragg scattering favors the formation of broad band gaps.

\section{CONCLUSION}

The proposed Matryoshka sonic crystal offers a viable solution to overcome the inherent dependence on spacing experienced with conventional sonic crystal designs. It has been discovered that such systems can form multiple resonance band gaps in the lower frequency region below that of Bragg formation. These resonance bands can be combined to form broad regions of attenuation either by selecting close acoustic resonances or further by tuning the structure to combine the characteristic Bragg band gap with the resonance band gaps.

The proposed six shell Matryoshka design is particularly suited for noise barrier applications. Although road traffic noise is essentially broad band in nature (due to a large number of very different vehicles that move at different velocities), it often has a defined maximum frequency. A study by Sandberg ${ }^{19}$ found that a multi-coincidence peak in the tyrenoise spectra is observed around $1000 \mathrm{~Hz}$. The simulation results obtained using a six shell concentric Matryoshka system demonstrates the active frequency range spans 400-1600 $\mathrm{Hz}$, providing decent levels of attenuation across this range. Moreover, the experimental results provided for the single Cshaped locally resonant sonic crystal, ${ }^{6}$ offer $\sim 25 \mathrm{~dB}$ of attenuation for applications as a noise attenuation solution.

\section{ACKNOWLEDGMENTS}

This work was supported by the UK Engineering and Physical Sciences Research Council (Grant No. EP/I029001/1).

\section{APPENDIX: PLANE WAVE EXPANSION METHOD}

The plane wave expansion (PWE) method is a commonly utilized numerical technique to calculate the band structures for phononic crystals. ${ }^{20,21}$ The PWE method can be applied to 
a phononic crystal with any solid scatterer but only infinite arrays can be modeled. The main technique of PWE is to expand the system parameter functions (density, speeds) and wavefunctions by plane waves in the wave equation in Fourier series. ${ }^{22}$ An infinite periodic array of scatterers can be modeled by applying the Floquet-Bloch theorem to the PWE.

The wave equation is

$$
\nabla \cdot\left[\frac{1}{\rho(\overrightarrow{\mathbf{r}})} \nabla p(\tilde{\mathbf{r}})\right]+\frac{\omega^{2}}{\rho(\tilde{\mathbf{r}}) c^{2}(\tilde{\mathbf{r}})} p(\tilde{\mathbf{r}})=0
$$

where $\rho(\tilde{\mathbf{r}})$ and $c(\tilde{\mathbf{r}})$ are the mass density and the sound speed, respectively; both are modulated by the periodic structures.

We can rewrite the wave equation with a definition of a scalar potential $\Phi(\tilde{\mathbf{r}}, t)$ such that $\rho \tilde{\mathbf{u}}=\nabla \Phi$ :

$$
\frac{1}{\rho c_{l}^{2}} \frac{\partial^{2} \Phi}{\partial t^{2}}=\nabla \cdot\left(\rho^{-1} \nabla \Phi\right),
$$

where $1 / \rho c_{l}^{2}$ is the longitudinal elastic constant.

According to Bloch's theorem, the solution of the sound pressure field has the Bloch form:

$$
p(\tilde{\mathbf{r}})=e^{i(\tilde{\mathbf{k}}) \cdot(\tilde{\mathbf{r}})-\omega t} \sum_{(\tilde{\mathbf{G}})} \phi_{\tilde{\mathbf{k}}}(\tilde{\mathbf{G}}) e^{i(\tilde{\mathbf{G}}) \cdot(\tilde{\mathbf{r}})},
$$

where $(\tilde{\mathbf{k}})$ is termed the Bloch wavevector, $(\tilde{\mathbf{G}})$ is the reciprocal lattice vector. The summation is made for all possible reciprocal vectors.

For periodic structures, both $\rho^{-1}$ and $\left(\rho c^{2}\right)^{-1}$ in the wave equation can be expanded by discrete plane waves as follows:

$$
\begin{aligned}
& \frac{1}{\rho(\tilde{\mathbf{r}})}=\sum_{(\tilde{\mathbf{G}})} \sigma(\tilde{\mathbf{G}}) e^{i(\tilde{\mathbf{G}}) \cdot(\tilde{\mathbf{r}})} \\
& \frac{1}{\rho(\tilde{\mathbf{r}}) c^{2}(\tilde{\mathbf{r}})} p(\tilde{\mathbf{r}})=\sum_{(\tilde{\mathbf{G}})} \eta(\tilde{\mathbf{G}}) e^{i(\tilde{\mathbf{G}}) \cdot(\tilde{\mathbf{r}})} .
\end{aligned}
$$

As $\rho(\tilde{\mathbf{r}})$ and $c(\tilde{\mathbf{r}})$ are known parameters, both $\sigma(\tilde{\mathbf{G}})$ and $\eta(\tilde{\mathbf{G}})$ can be determined from an inverse Fourier transform.

Substituting the Bloch form and the expanded wave equation back into the initial wave equation gives

$$
\begin{aligned}
& -\sum_{(\tilde{\mathbf{G}})}\left[\sigma\left((\tilde{\mathbf{G}})-\left(\tilde{\mathbf{G}}^{\prime}\right)\right)((\tilde{\mathbf{k}})+(\tilde{\mathbf{G}})) \cdot\left((\tilde{\mathbf{k}})+\left(\tilde{\mathbf{G}}^{\prime}\right)\right)\right. \\
& \left.\quad-\eta\left((\tilde{\mathbf{G}})-\left(\tilde{\mathbf{G}}^{\prime}\right)\right) \omega^{2}\right] \sigma_{(\tilde{\mathbf{k}})\left(\tilde{\mathbf{G}}^{\prime}\right)}=0 .
\end{aligned}
$$

Using a finite number $M$ of Fourier components in the expansion, an appropriate $M \times M$ matrix equation, $\Gamma$ can be solved:

$$
\sum_{\left(\tilde{\mathbf{G}}^{\prime}\right)} \Gamma_{(\tilde{\mathbf{G}}),\left(\tilde{\mathbf{G}}^{\prime}\right)} \phi_{\overrightarrow{\mathbf{k}}}\left(\tilde{\mathbf{G}}^{\prime}\right)=0 .
$$

The secular equation

$$
\begin{gathered}
-\operatorname{det}\left[\Gamma_{(\tilde{\mathbf{G}}),\left(\tilde{\mathbf{G}}^{\prime}\right)}\right]=\operatorname{det}\left[\sigma\left((\tilde{\mathbf{G}})-\left(\tilde{\mathbf{G}}^{\prime}\right)\right)((\tilde{\mathbf{k}})\right. \\
\left.+(\tilde{\mathbf{G}}))-\eta\left((\tilde{\mathbf{G}})-\left(\tilde{\mathbf{G}}^{\prime}\right)\right) \omega^{2}\right]_{(\tilde{\mathbf{G}}),\left(\tilde{\mathbf{G}}^{\prime}\right)}=0
\end{gathered}
$$

gives the dispersion relation between the frequency $\omega(k)$ and the wavevector $\tilde{k}^{22}$

For a two-dimensional phononic crystal system with a square lattice geometry, we have to define some variable in the PWE method. For such a system the cylinder material has density $\rho_{a}$ and it occupies a fraction $f$ of the background material with density $\rho_{b}$. Then,

$$
\begin{aligned}
\rho(\tilde{\mathbf{G}}) & = \begin{cases}\rho_{a}^{-1} f+\rho_{b}^{-1}(1-f) \equiv \overline{\rho^{-1}}, & \tilde{\mathbf{G}}=0, \\
\left(\rho_{a}^{-1}-\rho_{b}^{-1}\right) F(\tilde{\mathbf{G}}) \equiv \Delta \rho^{-1} F(\tilde{\mathbf{G}}), & \tilde{\mathbf{G}} \neq 0,\end{cases} \\
f(\tilde{\mathbf{G}}) & =A_{c}^{-1} \int d^{2} r e^{(-i \tilde{\mathbf{G}} \cdot r)} .
\end{aligned}
$$

The system comprises elastic rods with circular cross-section embedded in air. Because the system has circular scatterers the structure factor is defined in the PWE method as

$$
F(\tilde{\mathbf{G}})=2 f J_{1}\left(G r_{0}\right) /\left(G r_{0}\right)
$$

where $J_{1}$ is the Bessel function of the first kind. The square lattice configuration has a reciprocal lattice vector defined in the PWE method as

$$
\tilde{\mathbf{G}}=\left(\frac{2 \pi}{a}\right)\left(n_{x} x+n_{y} y\right) .
$$

A periodic lattice of steel cylinders in an air background is one of the most studied phononic crystal configurations, so this is a good basis to start from. For such a system, because the density contrast of steel and air $\rho_{s} / \rho_{a}$ is very large, the shear stress and transverse waves inside the steel cylinders will not make a significant contribution to the scattering of the acoustic waves in the air background. consequently the scaler acoustic wave equation is adequate to describe the scattering events of pressure waves at the steel cylinder interfaces, therefore the PWE method is sufficient for this system.

The eigenvalue problem is obtained by computing an acoustic wave equation with pressure to obtain the corresponding band structure of this system. The integers $n_{x}$ and $n_{y}$ were permitted to take values between -10 and +10 , providing 441 plane waves. This resulted in a good convergence.

\section{A. Conventional phononic crystal}

A conventional phononic crystal system comprising circular steel cylinders embedded in air, with lattice parameter $22 \mathrm{~mm}$ and a scatterer radius $6.5 \mathrm{~mm}$, is described in the density and shape functions in the PWE method. The dispersion relation is obtained by plotting frequency against the reduced wavevector. This will indicate any regions where

TABLE II. PWE material parameters for a phononic crystal system comprising steel scatterers embedded in air.

\begin{tabular}{lcc}
\hline \hline Material & Density $\left(\mathrm{kg} \mathrm{m}^{-3}\right)$ & Velocity of sound $\left(\mathrm{ms}^{-1}\right)$ \\
\hline Steel & 7800 & 6100 \\
Air & 1.2 & 343 \\
\hline \hline
\end{tabular}




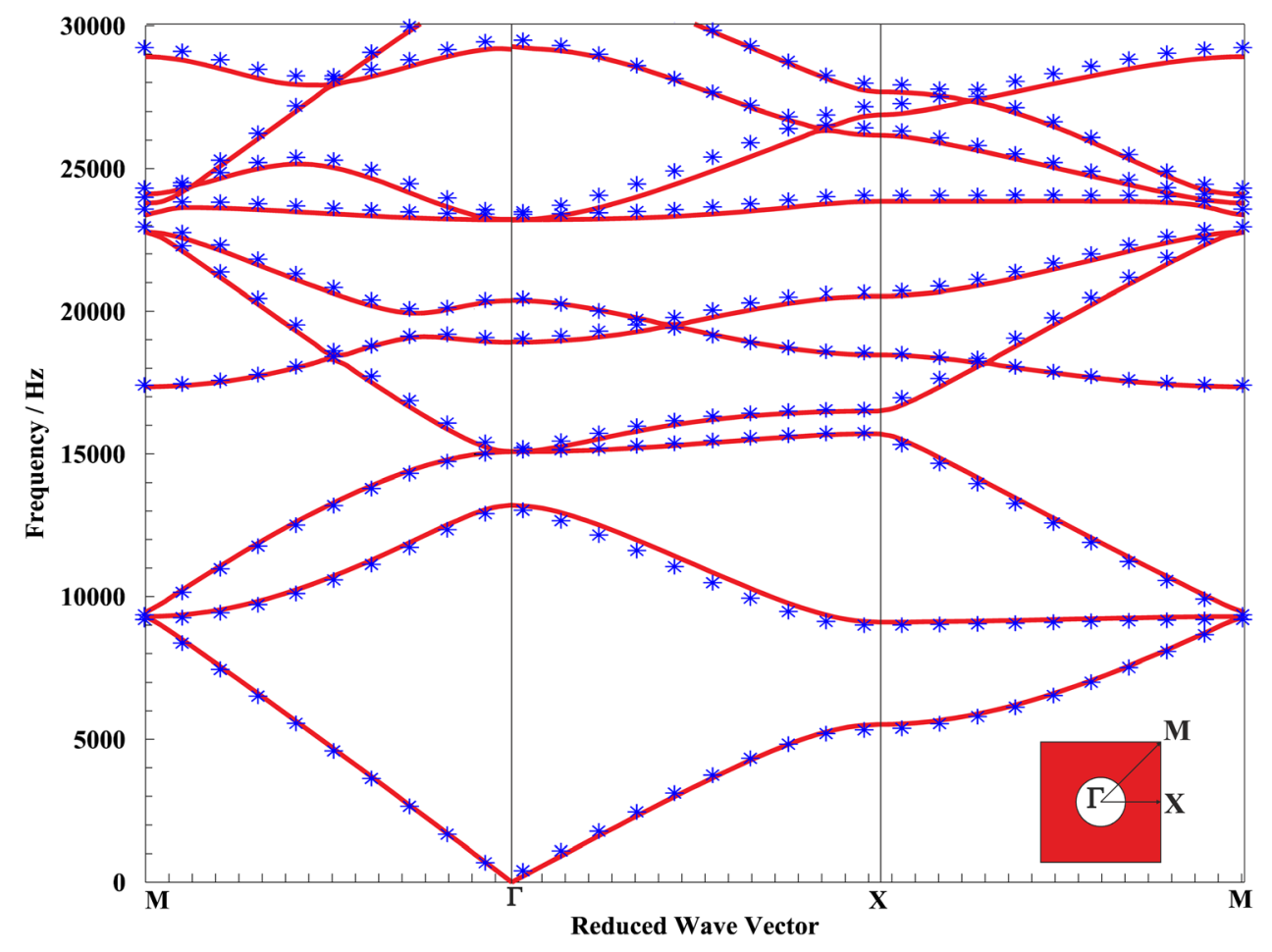

FIG. 13. (Color online) FEM computed band structure for a phononic crystal consisting of steel scatterers embedded in air $(r=6.5 \mathrm{~mm}, a=22$ $\mathrm{mm})$. The shading indicates the presence of a band gap. Included on the plot is the corresponding computed band structure from the PWE method (markers).

band gaps exist. The PWE material parameters are described in Table II. When coherent scattering occurs from equally spaced layers in a phononic crystal, the band gap opens up close to the first branch folding, i.e., at the border of the first Brillouin zone $k_{B Z}=\pi / a$. The dispersion in the vicinity of the band gap will be modified, but the center frequency of the band gap is approximately given by assuming linear dispersion and using $k_{B Z}$ :

$$
\omega=v k_{B Z}=v \frac{\pi}{a}
$$

which can be rearranged to give a simple relation to the center frequency of a Bragg band gap:

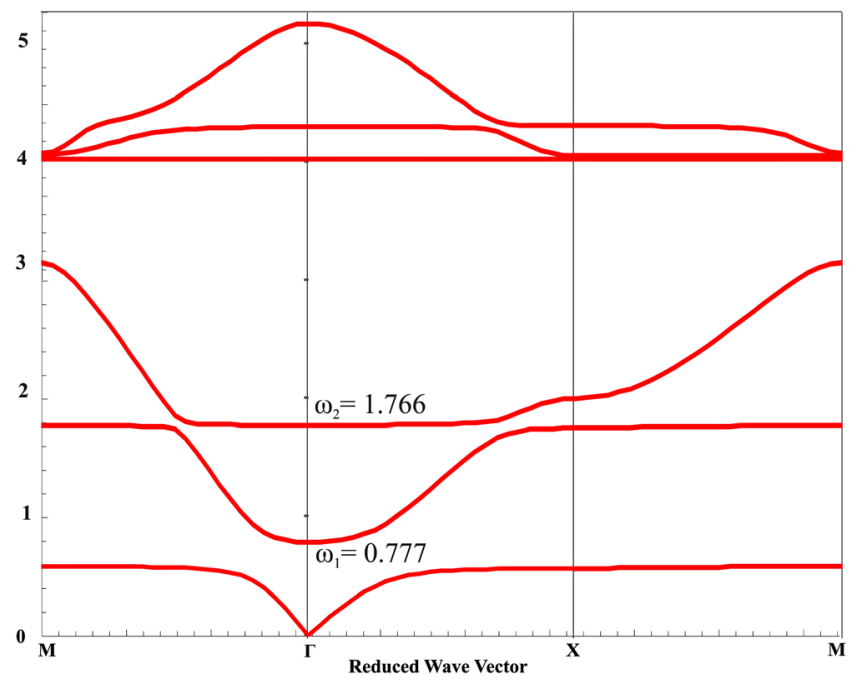

FIG. 14. (Color online) FEM computed band structure for multistructure described by Movchan et al. in Ref. (10), showing good agreement with calculated frequencies of the localized standing waves.

$$
f_{c}=\frac{v}{2 a}=\frac{343}{2 \times 0.022}=7795 \pm 20 \mathrm{~Hz} .
$$

Figure 13 shows the band structure (markers) for a phononic crystal system with a filling fraction of $f=0.274$. The shading in the figure indicates areas where band gaps are present. The inset in the figure is the first Brillouin zone. $\Gamma X$ refers to the $\left[\begin{array}{ll}1 & 0\end{array}\right]$ direction, and $\Gamma M$ the $\left[\begin{array}{ll}1 & 1\end{array}\right]$ direction, while $X M$ refers to the wavevector varying from [1 0 ] to [1 1] on the side of the Brillouin zone. It can be seen that toward the edges of the Brillouin zone that the dispersion is no longer linear with a curving of the bands and an opening of a band gap in the $\Gamma X$ direction with the first band gap extending from 5525 to $9125 \mathrm{~Hz}$, centered at $7325 \mathrm{~Hz}$. An acoustic band gap can be observed due to the low packing fraction, a complete acoustic band gap is not formed in all directions. Four additional band gaps are present in the first 10 eigenvalues (bands), the second spanning $12950-15000 \mathrm{~Hz}$, centered at $13975 \mathrm{~Hz}$, third spanning $16525-18500 \mathrm{~Hz}$, centered at $17513 \mathrm{~Hz}$, and last $20550-23250 \mathrm{~Hz}$, centered at $21900 \mathrm{~Hz}$. This is in excellent agreement with the results obtained by FEM shown in Fig. 13 (solid lines). Finally, using the finite element method, the band structure for multistructure described by Movchan et al. in Ref. 10 can be seen in Fig. 14, showing good agreement with calculated frequencies of the localized standing waves.

${ }^{1}$ M. Sigalas, M. S. Kushwaha, E. N. Economou, M. Kafesaki, I. E. Psarobas, and W. Steurer, "Classical vibrational modes in phononic lattices: Theory and experiment," Z. Kristallogr. 220, 765-809 (2005).

${ }^{2}$ R. Martinez-Sala, J. Sancho, J. V. Sanchez, V. Gomez, J. Llinares, and F. Meseguer, "Sound attenuation by sculpture," Nature 378, 241 (1995).

${ }^{3}$ J. V. Sanchez-Perez, D. Caballero, R. Martinez-Sala, C. Rubio, J. Sanchez-Dehesa, F. Meseguer, J. Llinares, and F. Galvez, "Sound attenuation 
by a two-dimensional array of rigid cylinders," Phys. Rev. Lett. 80, 5325-5328 (1998).

${ }^{4}$ J. V. Sanchez-Perez, C. Rubio, R. Martinez-Sala, R. Sanchez-Grandia, and V. Gomez, "Acoustic barriers based on periodic arrays of scatterers," Appl. Phys. Lett. 81, 5240-5242 (2002).

${ }^{5}$ P. Sheng, X. X. Zhang, Z. Liu, and C. Chan, "Locally resonant sonic materials," Appl. Phys. Lett. 289, 1734 (2000).

${ }^{6}$ L. Chalmers, D. Elford, F. Kusmartsev, and G. Swallowe, "Acoustic band gap formation in two-dimensional locally resonant sonic crystals comprised of helmholtz resonators," in Proceedings of the 32nd International Workshop on Condensed Matter Theories, Loughborough, 2010, Vol. 24, pp. 302; Int. J. Mod. Phys. B 23, 4234 (2009).

${ }^{7}$ X. Hu, C. T. Chan, and J. Zi, "Two-dimensional sonic crystals with helmholtz resonators," Phys. Rev. E. 71, 055601 (2005).

${ }^{8} \mathrm{~L}$. Y. Wu and L. W. Chen, "Wave propagation in a $2 \mathrm{~d}$ sonic crystal with a helmholtz resonant defect," J. Phys. D 43, 055401 (2010).

${ }^{9}$ S. Guenneau, A. Movchan, G. Petursson, and S. A. Ramakrishna, "Acoustic metamaterials for sound focussing and confinement," New J. Phys. 9, 399 (2007).

${ }^{10}$ A. B. Movchan and S. Guenneau, "Split-ring resonators and localized modes," Phys. Rev. B. 70, 125116 (2004).

${ }^{11}$ R. Sainidou and A. M. N. Stefanou, "Formation of absolute frequency gaps in three-dimensional solid sonic crystals," Phys. Rev. B 66, 212301 (2002).
${ }^{12}$ T. Still, W. Cheng, M. Retsch, R. Sainidou, J. Wang, U. Jonas, N. Stefanou, and G. Fytas, "Simultaneous occurrence of structure-directed and particle-resonance-induced phononic gaps in colloidal films," Phys. Rev. Lett. 100, 194301 (2008).

${ }^{13}$ L. Y. Wu and L. W. Chen, "The dispersion characteristics of sonic crystals consisting of elliptic cylinders," J. Phys. D 40, 7579 (2007).

${ }^{14} \mathrm{~V}$. Romero-Garcia and E. Fuster-Garcia, "Interferometric method of determining the refractive index of two-dimensional sonic crystals," Phys. Rev. B 75, 224305 (2007).

${ }^{15}$ L. Kinsler, Fundamentals of Acoustics, 4th ed. (Wiley, New York, 2000) (For our 2D case $V=\pi r^{2}$ ), p. 285.

${ }^{16}$ COMSOL MULTiPHYsics. Version 3.5a (Comsol Multiphysics, Ltd., 2007).

${ }^{17}$ T. T. Wu and Z. G. Huang, "Level repulsions of bulk acoustic waves in composite materials," Phys. Rev. B 70, 214304 (2004).

${ }^{18}$ W. A. Harrison, Solid State Theory (Dover Publications, New York, 1980), p. 554.

${ }^{19} \mathrm{U}$. Sandberg, "The multi-coincidence peak around $1000 \mathrm{~Hz}$ in tyre/road noise spectra," in Proceedings of Euronoise, Naples, Italy (2005), p. 498.

${ }^{20}$ M. M. Sigalas and E. N. Economou, "Elastic and acoustic wave band structure," J. Sound Vib. 158, 377 (1992).

${ }^{21}$ M. S. Kushwaha, "Theory of acoustic band structure of periodic elastic composites," Phys. Rev. B. 49, 2313-2322 (1994).

${ }^{22}$ T. Miyashita, "Sonic crystals and sonic wave-guides," Meas. Sci. Technol. 16, R47-R63 (2005) 